\title{
An Unusual Second Rupture of the Achilles Tendon: A case report
}

\author{
by Oladejo A. Olaleye, MRCS Ed., DOHNS, MBBS ${ }^{1} \rrbracket$, Helmut Zahn, FRCS (Tr\&Ortho) ${ }^{2} \rrbracket$
}

The Foot \& Ankle Journal 1 (12): 3

This case report describes an unusual second rupture sustained after conservative treatment of an initial Achilles tendon rupture at the musculotendinous junction. The initial injury was successfully managed conservatively with an equinus cast for 6 weeks. The patient developed a tendonitis 10cm below the initial rupture and subsequently sustained a second traumatic rupture of the Achilles tendon at a new site after tripping on a curb. The patient had no known systemic diseases and was not on steroid or fluoroquinolone therapy. The site of this second rupture was repaired surgically using an open technique without any long term complications.

Key words: Achilles tendon, rupture, traumatic

Accepted: November, 2008 Published: December, 2008

This is an Open Access article distributed under the terms of the Creative Commons Attribution License. It permits unrestricted use, distribution, and reproduction in any medium, provided the original work is properly cited. @The Foot \& Ankle Journal (www.faoj.org)

\section{Case report}

A 37 year-old male, dog-handler from the police force was chasing a suspect when he experienced acute pain to the right posterior leg in the Achilles region. He was unable to ambulate after the initial injury. No previous leg injuries or tendinitis were reported. The patient did not report any history of taking corticosteroids or fluoroquinolone antibiotics. Clinically, the patient had a positive Simmond's test and rupture of the Achilles tendon was isolated to the musculo-tendinous junction.

\footnotetext{
Address correspondence to: Olaleye MRCS Ed., DOHNS, MBBS Trauma and Orthopaedic Surgery Department William Harvey Hospital, Ashford

Kent, United Kingdom. TN24-OLZ

Email: dejolaleye@yahoo.com

${ }^{1}$ CT1 Trauma and Orthopaedic Surgery, William Harvey Hospital. Ashford, Kent. UK.

${ }^{2}$ Consultant, Trauma and Orthopaedic Surgery, William Harvey

Hospital, Ashford, Kent. UK.
}

A clinical diagnosis of Achilles rupture was rendered. No magnetic resonance imaging (MRI) was initially performed and the patient was managed conservatively in an equinus cast for 6 weeks and then in an adjustable boot to bring the foot to ankle range of motion to 90 degrees over the following 6 weeks. The adjustable boot was initially put in 20 degrees of dorsiflexion for 3 weeks then adjusted to 90 degrees over the following course of treatment.

The adjustable boot was removed and he managed to walk without crutches. The patient had regained total range of motion of the ankle with no gap or tenderness along the Achilles tendon and good plantarflexion power. 


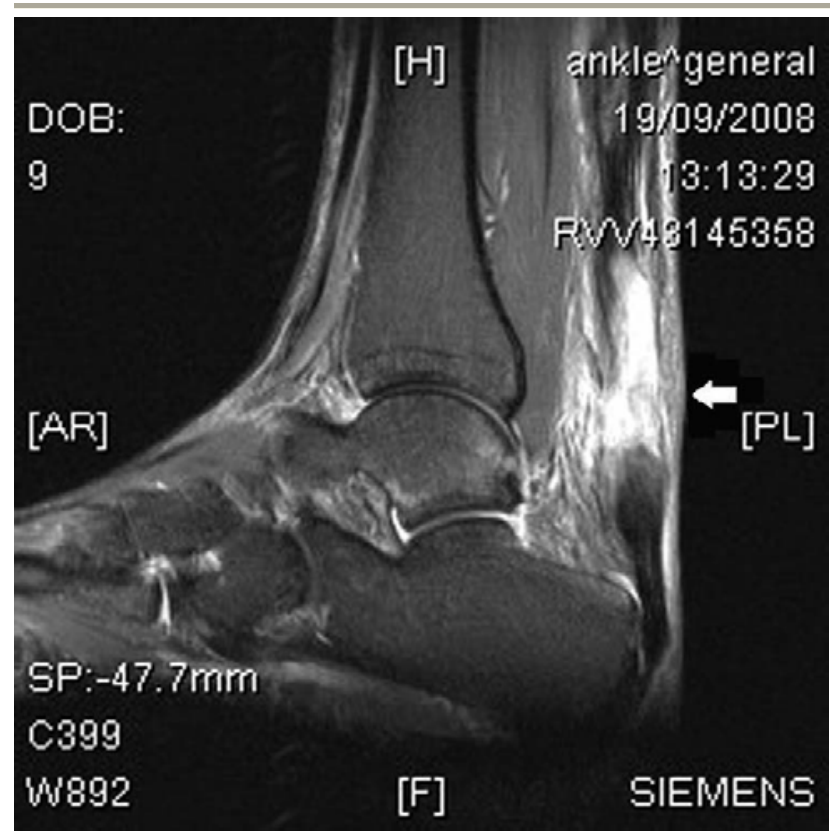

Figure 1 Region of second rupture as identified on MRI (arrow). More proximally, the initial injury can be seen at the musculo-tendinous junction.

\section{Reinjury}

He subsequently developed a painful lump in his Achilles tendon $10 \mathrm{~cm}$ inferior to the initial rupture location. Clinically, he had a fusiform swelling of the tendon which was slightly fluctuant and tender to palpation. He was placed on a course of anti-inflammatory medication and referred to physiotherapy for ultrasound. To confirm the diagnosis, an MRI scan was scheduled. During this period, the patient sustained a second injury to the same tendon while waiting for the MRI scan. The patient had been walking on the road with a group of friends when he tripped on a curb and felt a sharp pain along his right Achilles tendon. MRI confirmed a second injury more inferior to the original site of injury. (Fig. 1)

MRI of the site of injury revealed an abnormally high signal change and thickening within a somewhat retracted Achilles tendon $3 \mathrm{~cm}$ above its insertion as well as at the musculo-tendinous junction of the gastrocnemius muscle.

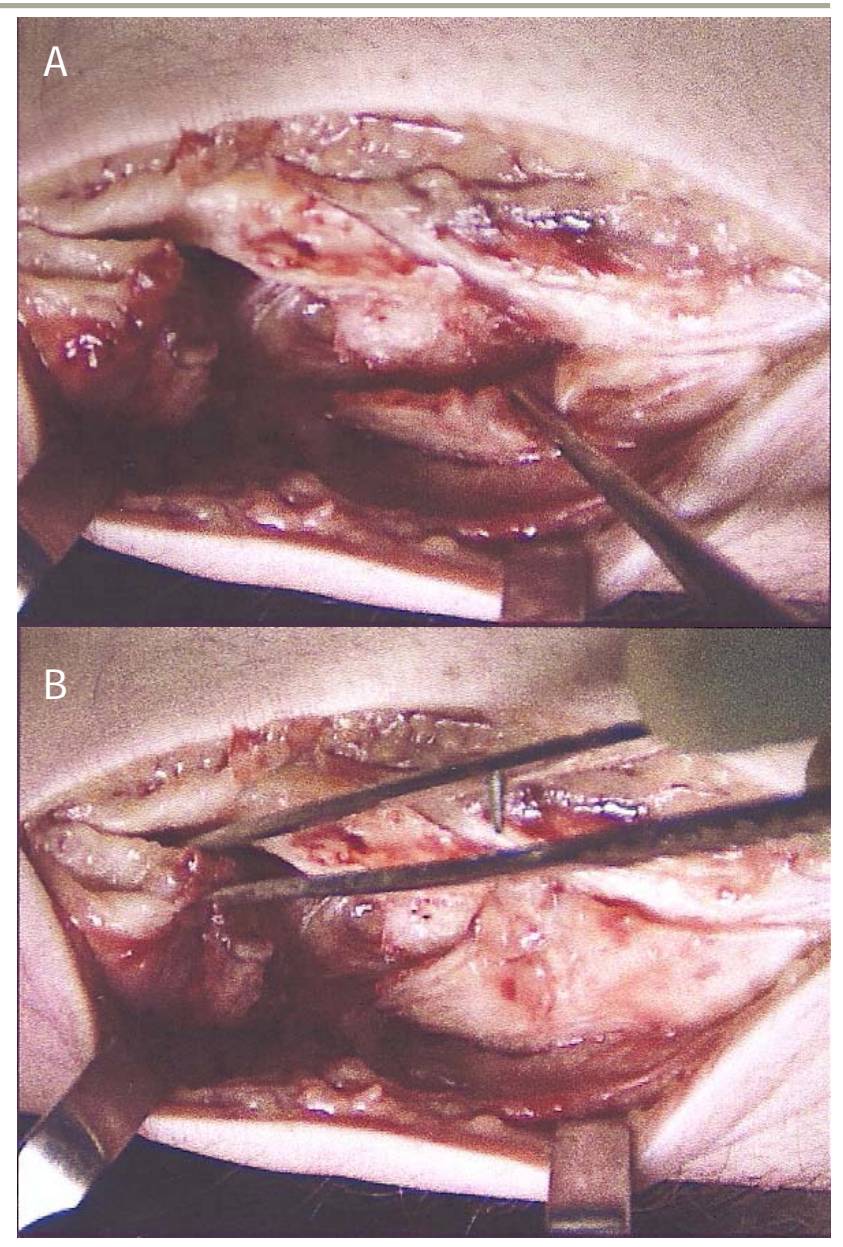

Figure 2A and B Intra-operatively, substantial attenuation of reinjury is identified with a forcep holding distal end of achilles tendon rupture, $3 \mathrm{~cm}$ above its calcaneal insertion (A) The proximal end of the ruptured tendon is also identified with a forcep. (B)

This was consistent with a full-thickness, highgrade tear or rupture of the Achilles tendon at the musculo-tendinous junction. Intra-tendinous edema associated with the presence of fluid was located within and about the Achilles tendon suggesting the presence of intra-tendinous hematoma. There was also swelling of the posterior surrounding subcutaneous soft tissues.

There was little fluid noted within the retrocalcaneal bursa or along the lower aspect of the Achilles tendon about $2.5 \mathrm{~cm}$ from its insertion. 


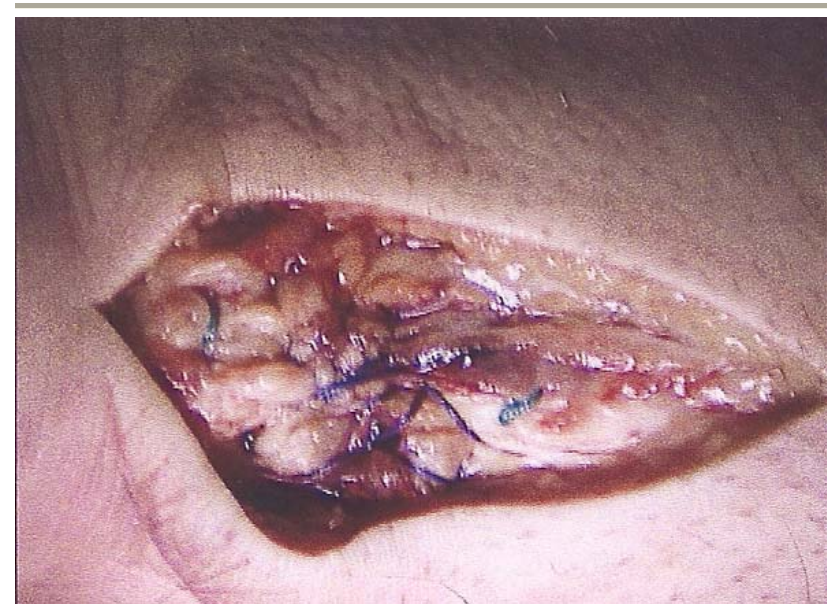

Figure 3 Repair after closure of the Achilles paratenon.

A small region of marrow edema was noted in the posterior aspect of the talus. The tibia and fibula were intact and no fracture of ankle was reported.

On his second presentation to the hospital, there was a palpable gap to the Achilles tendon with a positive Simmond's test. At this time, he was scheduled for primary surgical repair of the tendon.

Intraoperatively, the patient had a general anaesthetic, positioned in a prone position with tourniquet applied. The incision was limited to the second rupture site and the initial injury was not visualised as it was at the musculotendinous junction. The tendon was exposed demonstrating the second rupture with obvious complete rupture $3 \mathrm{~cm}$ above the calcaneus with high attenuation. (Figs. 2A and B)

The ruptured edges were identified and reapposed by a modified Kessler locking core stitch using No.5 Ethibond and a running suture using No.1 Vicryl. (Fig. 3) Postoperatively, he was placed in an equinus cast with DVT (deep vein thrombosis) prophylaxis and gradual rehabilitation with good recovery.

\section{Discussion}

Achilles tendon rupture is a complete disruption of the Achilles tendon most commonly affecting 30-50 year old males with the rupture about 4$5 \mathrm{~cm}$ above the calcaneus (a zone of poor blood flow in the tendon). ${ }^{7}$ There is a male:female predisposition of 6:1. Predisposing factors include chronic tendonitis or tendinopathy and prior cortisone injection treatments. The majority of these injuries occur during some athletic event requiring forceful push-off of the foot. Other associating risk factors include gout, patient with blood type $\mathrm{O}$, systemic lupus erythematosus, rheumatoid arthritis, patients on steroid medication and fluoroquinolone-type antibiotics. ${ }^{7}$

Achilles tendon rupture is often characterized by an acute onset of pain in the distal posterior portion of the lower leg. Patients often think they have been kicked, cut or hit in the back of the leg with subsequent difficulties ambulating or putting weight down on the affected foot.

Pathophysiological theories include chronic degeneration of the tendon and failure of the inhibitory mechanism of the musculotendinous unit. ${ }^{5}$ Some believe that it may be due to the whipping action or bowstring effect caused by ankle pronation, or by the Achilles tendon's relatively weak blood supply. Others consider the cause to be the combination and frequency of eccentric shortening when the heel hits the ground followed rapidly by concentric contraction when the toes push off.'

There is obvious leg swelling with a palpable defect in the Achilles tendon and a positive Thompson's test. The Thompson's test usually reveals absence of plantar flexion on squeezing the calf with the patient lying in the prone position. 
The diagnosis is often made clinically. Plain radiographs, ultrasonography, and MRI can also be useful to confirm the diagnosis. The MRI is the gold-standard for diagnosing acute Achilles tendon ruptures and can also confirm the diagnosis of a partial rupture which may not be clinically apparent.

Achilles tendon injuries can occur in different locations along the Achilles tendon. Tennis leg is a rupture between the Achilles tendon and the gastrocnemius. Achilles tendinosis, Achilles tendonitis, Achilles tenosynovitis, Achilles tendon rupture and medicine side effects are most often felt an inch or two above the heel. Insertional Achilles tendinosis, tendonitis, and tenosynovitis occur where the Achilles tendon and heel connect. Achilles tendon laceration or crushing could occur anywhere along the Achilles tendon. ${ }^{9}$

Achilles tendon ruptures can be managed conservatively or operatively and several methods have been employed with varying results. Lower re-rupture rates and slightly improved strength and functional ability may be expected with surgical repair. The rate of minor surgical complications is higher than that of non-operative treatment. ${ }^{5}$ With careful attention to the surgical wound and patient compliance to post-operative rehabilitation protocols, operative repair of acute Achilles tendon ruptures is a reliable treatment for active patients. ${ }^{8}$ Three partial Achilles tendon reruptures and one complete rupture were documented in a series of 74 patients that had operative repair. ${ }^{8}$ A known benefit of surgical repair is the decreased re-rupture rate. One study showed a $4 \%$ re-rupture rate for operative repair compared to an $8 \%$ re-rupture rate for conservative management. ${ }^{6}$ Re-rupture rates of $1.4 \%$ and $13.4 \%$ for surgical and conservative management respectively have been reported. ${ }^{1}$ A meta-analysis found re-rupture rates of $1 \%$ and $18 \%$ for surgical and conservative repair. ${ }^{3}$ There were no re-ruptures for 44 patients treated surgically as opposed to 9 of the 44 for those treated conservatively. ${ }^{2}$
Recent studies have suggested better outcomes with early postoperative functional rehabilitation. An unusual type rupture, where the Achilles tendon was ruptured in two places requiring several innovative techniques to repair has been described. ${ }^{4}$

In our institution, we prefer operative approach with early mobilisation in an adjustable air cast boot. The usual regime is to apply a dorsal slab until the wound has healed. An adjustable air cast boot is then applied starting at 45 degrees of equinus aiming to reach 90 degrees by 6 weeks, at which point, weight bearing is allowed. Passive dorsiflexion is continued until range of movement equals contralateral side. The air cast is discarded 3 months after surgical repair.

\section{Conclusion}

This case report highlights an unusual second rupture of the Achilles tendon at a different site on the same tendon following an initial traumatic rupture at the musculotendinous junction. This is a rarely reported injury in the literature. This previously fit and healthy patient had a traumatic rupture of his right Achilles tendon at the musculotendinous junction which was treated conservatively in an equinus cast. He subsequently developed a tendonitis and then traumatic rupture at a site $10 \mathrm{~cm}$ below the initial rupture on the same tendon. This was treated with open surgical repair and rehabilitation.

In conclusion, the presentation of this case study has highlighted the potential role for routine MRI scanning to assess Achilles tendon ruptures and identification of risk for a second rupture. 


\section{References}

1. Cetti R, Christensen SE, Ejsted R, Jensen NM, Jorgensen $\mathrm{U}$. Operative versus non-operative treatment of Achilles tendon rupture. A prospective randomized study and review of the literature. Am J Sports Med. 21 (6): 791 - 799, 1993.

2. Inglis AE, Scott WN, Sculco TP, Patterson AH. Ruptures of the tendo Achilles: an objective assessment of surgical and non-surgical treatment. J Bone Joint Surg 58A (7): 990 - 993, 1976.

3. Kellam JF, Hunter GA, McElwain JP. Review of the operative treatment of Achilles tendon rupture. Clin Orthop 201: 80 - 83, 1985.

4. Kuwada GT. A severe acute Achilles rupture and repair. J Foot Ankle Surg 34 (3): 262 - 5, 1995.

5. Leppilahti J, Orava S. Total Achilles tendon rupture. A review. Sports Med 25(2): 79 - 100, 1998.

6. Nistor L. Surgical and non-surgical treatment of Achilles tendon rupture: a prospective randomized study. J Bone Joint Surg 63A (3): $394-399,1981$.

7. Saglimbeni AJ \& Fulmer CJ. Achilles tendon injuries and tendonitis. Emedicine, 2008.

8. Strauss EJ, Ishak C, Jazrawi L, Sherman O, Rosen J. Operative treatment of acute Achilles tendon ruptures: an institutional review of clinical outcomes. Injury 38(7): 832 838, 2007.

9. Everything about Achilles tendons (2004-2006). Online, accessed 24th November 2008. 